\title{
Enfermedad cardíaca y embarazo
}

\author{
Hernán Cortés Yepes*
}

\section{RESUMEN}

La enfermedad cardíaca durante el embarazo continua siendo una importante causa de morbi-mortalidad materna y fetal, debido al enmascaramiento y en algunas situaciones al empeoramiento de estas entidades.

El manejo de la embarazada cardiópata, requiere el entendimiento de los cambios fisiológicos de la gestación y debe ser llevado a cabo por un equipo multidisciplinario.

Se presenta una revisión de la etiología, consejería prenatal y diagnóstico y se hacen recomendaciones de manejo general, vía del parto y profilaxis contra la endocarditis, con el fin de mejorar la atención de estas pacientes.

PALABRAS CLAVES: Embarazo, enfermedad cardíaca, consejería prenatal, manejo general, vía del parto, profilaxis antimicrobiana.

\section{SUMMARY}

Cardiac disease in pregnancy continue being an important cause of both maternal and fetal morbidity and mortality due to hiding and deterioration of these conditions.

The management of the pregnant women with a cardiac disease requires a know ledge of the physiological vhanges during pregnancy and it must be carried out by a multidisciplinary team.

One presents a review of the ethiology, preconception counseling and diagnosis and one does recommendations about the general management, mode of delivery and prophylaxis against the bacterial endocarditis in order to improve patient care.

KEY WORDS: Pregnancy, Cardiac disease, Preconception counseling, General management, Mode of delivery, Antibacterial prophylaxis.

\section{Incidencia y etiología}

La enfermedad cardiovascular complica el uno por ciento de todos los embarazos, ocupando el tercer puesto en la mortalidad materna y la primera causa de origen no obstétrico en los países industrializados, siendo responsable del $5.6 \%$ de las muertes maternas en Estados Unidos entre 1987 y 1990.

Actualmente en los países desarrollados, la principal causa de enfermedad cardiovascular en el embarazo es de origen congénito, debido a la casi desaparición de la fiebre reumática y a la mejoría en la atención y manejo quirúrgico de estas pacientes. Otras causas aunque menos frecuentes incluyen, las cardiopatías hipertensiva, isquémica, sifilítica y las cardiomiopatías, entre otras (1-2).

En nuestro medio, se han realizado pocos estudios sobre este tema. En el hospital San Vicente de Paúl, durante 1998, Vásquez y Cols., encontraron 64 pacientes con una edad promedio de 24.8 años remitidos con diagnóstico y/o

Residente de II año de Ginecología y Obstetricia. Departamento de Ginecología y Obstetricia. Universidad de Antioquia. Medellín Colombia. sospecha de enfermedad cardíaca y embarazo, de las cuales un $31 \%$ tenían una cardiopatía de origen reumático, 26\% una cardiopatía congénita (29\% CIV, $23 \%$ CIA, 23\% PVM.), $15 \%$ con alteraciones del ritmo y $26 \%$ con soplos funcionales. (3). Se demuestra entonces, la alta incidencia de fiebre reumática y sus secuelas en nuestra población, siendo aun esta la principal causa de enfermedad cardíaca en este grupo de edad.

Diagnóstico de enfermedad cardíaca en el embarazo.

El diagnóstico de la enfermedad cardíaca durante el embarazo es un reto para el médico, ya que los cambios hemodinámicos fisiológicos de este periodo simulan los signos y síntomas de una cardiopatía y hacen más difícil su detección.

En la embarazada es común la disnea, la fatiga, la ortopnea y ocasionalmente lipotimia y al examen físico se encuentran soplos sistólicos funcionales en el $95 \%$ de las pacientes, además es común la aparición de un tercer sonido (S3), el desdoblamiento del segundo ruido, edema de miembros inferiores, crépitos en bases pulmonares y distensión de los vasos del cuello. 
Sin embargo algunos síntomas y signos son indicadores de enfermedad cardíaca durante el embarazo. (Tabla 1).

El embarazo puede también alterar los exámenes de laboratorio, es así como en el electrocardiograma se encuentra desviación del eje cardíaco hacia la izquierda de aproximadamente 15 grados, con alteraciones del segmento S-T en DIII y aVF, y ocasionalmente pequeñas ondas Q asociadas a inversión de la onda $\mathrm{T}$, en estas mismas derivaciones, además las extrasístoles y la taquicardia supraventricular son mas frecuentes en el embarazo (4).

En la ecocardiografía, se evidencia una leve dilatación ventricular, asociada a insuficiencia tricuspidea y pulmonar y en menor frecuencia mitral, debido a la dilatación del anillo valvular, con aumento del tamaño del tracto de salida del ventrículo izquierdo (5).

\section{Consejería prenatal}

Las mujeres que padecen una enfermedad cardíaca y que desean un embarazo, deben recibir asesoría de un equipo multidisciplinario, para determinar la severidad y posibles implicaciones de este; Sabiendo que algunas patologías son curables como la CIA y el ductus persistente, entre otras y en otros casos la enfermedad puede ser mejorada como en la patología mitral, la estenosis aórtica, la CIV y algunas malformaciones congénitas, se debe aconsejar el tratamiento previo y esperar al menos un año antes de quedar en embarazo.

Sin embargo, en otras condiciones el embarazo esta contraindicado, debido al alto riesgo de mortalidad materna y fetal, como ocurre en el síndrome de Eisenmenger, la hipertensión pulmonar primaria y el síndrome de Marfá con compromiso aórtico, con tasa de mortalidad de un 30-70\% (4), por esto en países donde es permitido el aborto, este se aconseja en estas pacientes o el manejo intensivo por un equipo multidisciplinario, con hospitalización durante todo el embarazo cuando no lo aceptan.

Es de anotar que los bebes de madres con cardiopatía congénita tiene un mayor riesgo de malformaciones cardíacas, como en el caso de la CMP hipertrófica, que se transmite de manera autosómica dominante en el 50\% de los casos (1) o en la CMP dilatada, con patrones de transmisión variables y con incidencia de $7-20 \%(4,6)$. Pero también se ha encontrado una mayor incidencia de malformaciones no cardíacas, abortos, RCIU y mortinatos.

Además del riesgo al que se somete el feto por los diferentes medicamentos que ingiere la madre como los

Tabla 1

\begin{tabular}{|l|l|}
\hline SINTOMAS & SIGNOS \\
\hline $\begin{array}{l}\text { Disnea severa } \\
\text { Síncope con el ejercicio } \\
\text { Disnea paroxística nocturna } \\
\text { de grado 3 }\end{array}$ & Cianosis \\
Hemoptisis & Soplo diastólico sistólico mayor \\
Dolor precordial con el & Arritmia persistente \\
ejercicio & Hipocratismo digital \\
\hline
\end{tabular}

IECA y la warfarina entre otros. (Tabla 2) y el aunque menor riesgo de algunos exámenes de laboratorio (evaluación con radionucleótidos) y medidas terapéuticas que deban ser tomadas como la cardioversión, la valvulotomía, etc.

\section{Adaptaciones cardiovasculares al embarazo}

El manejo de la embarazada cardiópata, requiere el entendimiento de los cambios fisiológicos de la gestación, el parto y el puerperio, ya que estos pueden alterar la presentación y el curso de cada entidad.

El volumen sanguíneo se incrementa desde el primer trimestre hasta un $40-50 \%$ a la semana 30 , permaneciendo estable hasta el término, con un aumento del $20-40 \%$ de la masa eritrocitaria y de aproximadamente $50-60 \%$ en el volumen plasmático, que produce la denominada anemia fisiológica del embarazo, que requiere tratamiento si la hemoglobina es menor de $11 \mathrm{y}$ el hematocrito menor de 33; Este aumento, es debido a los cambios en el sistema renina angiotesina aldosterona, que aumenta junto con los niveles de estrógenos y progestágenos.

Hacia el término, se produce retención de agua en el tejido extracelular de aproximadamente 1 a 2 litros y de hasta 5 litros en las pacientes con edema. El edema, se presenta en el 50-80\% de las embarazadas.

El gasto cardíaco aumenta durante las 10 primeras semanas del embarazo y alcanza un 30-45\% a la semana 20, pero hacia el término este es variable, dependiendo de la posición materna, ya que el útero aumentado de tamaño disminuye la precarga en la posición supina, al interferir con el retorno venoso de las piernas.

La frecuencia cardíaca también aumenta de 10-20 latidos por minuto: Sin embargo a pesar del aumento del gasto cardíaco, la presión arterial tiende a disminuir, situación que obedece a la notable caída de la resistencia vascular sistémica, debido a la circulación uterina (de baja resistencia) y al efecto vascular de las hormonas placentarias.

La evaluación ecocardiográfica indica un aumento en la fracción de eyección de un 10-15 \% y de la velocidad de acortamiento de la fibra miocárdica, probablemente debido al aumento en la precarga y a la disminución de la postcarga (disminución de la resistencia vascular sistémica), no se ha demostrado que los cambios hormonales aumenten de manera directa la contractilidad cardíaca.

Durante el trabajo de parto y el parto, al corazón se le impone una mayor demanda debido al aumento del retorno venoso y del tono simpático, producidos por la contracción uterina y en respuesta al dolor que esta produce, aumentando el gasto cardíaco un $20 \%$ mas durante cada contracción.

En el postparto, la separación de la placenta y la involución uterina producen una "autotransfusión" de $500 \mathrm{mI}$ de sangre; Además la pérdida sanguínea, la excreción del volumen intra y extracelular aumentados y el inicio de la lactancia, hacen de este periodo uno de los más vulnerables para la descompensación hemodinámica.

El embarazo también se asocia con un estado de hipercoagulabilidad, debido a la disminución de la proteína S libre y de la actividad fibrinolítica del plasma, el aumento del fibrinógeno en un $50 \%$ y de los factores de la coagulación (II , VII, VIII, IX y X), además del estasis 
Tabla 2

POSIBLES EFECTOS ADVERSOS DE DROGAS CARDIOVASCULARES EN EL FETO

\begin{tabular}{|c|c|c|}
\hline MEDICAMENTO & EFECTOS ADVERSOS & $\begin{array}{l}\text { SEGURIDAD } \\
\text { CATEGORIA(FDA) }\end{array}$ \\
\hline Warfarina & $\begin{array}{l}\text { Hemorragia fetal in útero, } \\
\text { Embriopatía, malformaciones del SNC. }\end{array}$ & $\begin{array}{l}\text { No segura } \\
\mathrm{X}\end{array}$ \\
\hline Heparina & No reportado & Seguro \\
\hline Digoxina & Bajo peso al nacer & Seguro \\
\hline Quinidina & $\begin{array}{l}\text { Trombocitopenia, a dosis tóxicas } \\
\text { parto prematuro y daño del } 8^{\circ} \text { par craneal. }\end{array}$ & $\begin{array}{l}\text { Seguro } \\
\mathrm{C}\end{array}$ \\
\hline Procainamida & No reportado & Seguro \\
\hline Disopiramida & Puede desencadenar actividad uterina. & $\mathrm{C}$ \\
\hline Lidocaina & Acidosis fetal, depresión del SNC. & Seguro. \\
\hline Propafenona & No reportado & $\mathrm{C}$ \\
\hline Adenosina & No reportado & Seguro \\
\hline Amiodarona & RCIU, hipotiroidismo. & $\begin{array}{l}\text { No seguro } \\
\mathrm{C}\end{array}$ \\
\hline Bloqueadores canales de calcio & Sufrimiento fetal por hipotensión materna. & $\mathrm{C}$ \\
\hline Beta bloqueadores & $\begin{array}{l}\text { RCIU, mal adaptación neonatal } \\
\text { (hipoglicemia, apnea, bradicardia) } \\
\text { pueden iniciar actividad uterina. }\end{array}$ & $\begin{array}{l}\text { Seguro } \\
\mathrm{C}\end{array}$ \\
\hline Hidralazina & $\begin{array}{l}\text { Se ha reportado trombocitopenia y } \\
\text { Leucopenia }\end{array}$ & $\begin{array}{l}\text { Seguro } \\
\mathrm{C}\end{array}$ \\
\hline $\begin{array}{l}\text { Nitroprusiato de } \\
\text { sodio }\end{array}$ & Toxicidad por tiocianatos. & $\begin{array}{l}\text { Potencialmente } \\
\text { inseguro. }\end{array}$ \\
\hline Nitratos & Bradicardia & $\mathrm{C}$ \\
\hline $\begin{array}{l}\text { Inhibidores de } \\
\text { la ECA }\end{array}$ & $\begin{array}{l}\text { Defectos en la osificación del cráneo } \\
\text { RCIU, bajo peso al nacer, anemia, } \\
\text { oligoamnios, insuficiencia renal, } \\
\text { ductus persistente y muerte. }\end{array}$ & No seguro \\
\hline Diuréticos & $\begin{array}{l}\text { Hipoperfusión placentaria, bradicardia } \\
\text { trombocitopenia, hiponatremia }\end{array}$ & $\begin{array}{l}\text { Potencialmente } \\
\text { no seguro. C }\end{array}$ \\
\hline
\end{tabular}

Adaptado de Bonow et al. ACC/AHA task force report. JACC vol. 32 \#5. 1998:1486 y Shabetai, R. Cardiac disease en Creasy/Resnik. Maternal fetal medicine. $4^{\text {th }}$ edition W.B saunders company. p. 798-799. 
venoso y el daño vascular que se produce en el momento del parto.

El estado cardiovascular retorna a lo normal a la segúnda semana postparto $(4-5,7)$.

\section{Manejo general}

Aunque las diversas entidades cardíacas tienen un manejo específico y no se puede generalizar, algunas recomendaciones sirven de pautas de manejo.

La embarazada cardiópata debe realizar un control prenatal estricto, con un equipo multidisciplinario que incluya el cardiólogo, el obstetra y el anestesiólogo entre otros; Se debe estar atento a los signos y síntomas de descompensación, como el aumento en la frecuencia e intensidad de la disnea, la fatiga, la aparición de crépitos pulmonares asociados a tos, la hemoptisis y la taquicardia etc., ya que estos son indicadores de insuficiencia ventricular.

Se debe aconsejar el reposo, con el fin de disminuir la actividad física que demanda un mayor gasto al corazón el que se encuentra en su máxima capacidad; algunas pacientes deben permanecer en cama, principalmente durante el último trimestre.

En la dieta, se debe disminuir la ingesta de sodio para prevenir la excesiva acumulación de líquido y aumentar las calorías y vitaminas principalmente de hierro.

Se debe estar atento para prevenir y tratar las enfermedades que puedan descompensar a la paciente, como las infecciones principalmente de tipo respiratorio, evitando el contacto con personas con gripe y recibiendo vacunación, además prohibir el uso de cigarrillo y corregir la anemia con suplementos de hierro y ácido fólico.

Durante el trabajo de parto y el parto, se debe realizar una monitorización adecuada principalmente de tipo clínico, vigilando la frecuencia cardíaca y respiratoria, pero en ocasiones se requiere la monitorización invasiva para optimizar la hidratación y valorar objetivamente el gasto cardíaco.

No se debe exceder la administración de líquidos endovenosos, antes se recomienda mantenerlas "secas" con infusión de líquidos a $75 \mathrm{ml} / \mathrm{hr}$. (8)

La paciente, debe estar en decúbito lateral izquierdo y en algunas situaciones con oxígeno.

Se debe manejar adecuadamente el dolor, por las consecuencias adversas de este, se prefiere la analgesia epidural temprana, pero teniendo cuidado de la hipotensión que podría ser mal tolerada por la paciente.

En algunas entidades, como la estenosis aórtica, la hipertensión pulmonar y los shunts, se prefiere el uso de opiáceos sobre los anestésicos, ya que la vasodilatación que estos producen disminuye la precarga con empeoramiento de estas condiciones.

En el postparto se debe mantener una estrecha vigilancia pues es el periodo de mayor riesgo de descompensación, por la distribución del flujo; estas pacientes deben permanecer sentadas para disminuir el retorno venoso de los miembros inferiores y en algunas situaciones se deben usar diuréticos.

\section{Vía del parto}

Se prefiere el parto vaginal, dejando la cesárea para las indicaciones obstétricas, se recomienda el uso de fórceps, con el fin de disminuir el esfuerzo materno por el pujo y acortar el expulsivo.

Sin embargo, algunos autores sugieren que la cesárea es preferible al parto vaginal en algunas situaciones como en la coartación de aorta, el síndrome de Marfán, el síndrome de Ehlers Danlos y la aortitis de Takayasu, ya que durante el trabajo de parto y el parto, se presentan aumentos intermitentes en el gasto cardíaco y la presión arterial que pone en riesgo de disección aórtica a estas pacientes.

De igual manera en las pacientes con cardiopatía congénita cianosante severa, el trabajo de parto aumenta el shunt de derecha a izquierda, condición que agrava la hipoxemia fetal.

En condiciones como el síndrome de Eisenmenger, cuando el embarazo no se lleva al término, la vía vaginal podría tener mayor riesgo para un bebe prematuro y sometido a un stress crónico; además es más fácil planear la terminación de un embarazo por cesárea que por la vía vaginal.

Estos mismos autores aseguran que la cesárea con anestesia general permite mantener una situación cardiovascular estable, mejor oxigenación e hidratación disminuyendo el esfuerzo materno y mejorando la situación fetal (9).

\section{Profilaxis antimicrobiana}

La asociación americana del corazón no recomienda la profilaxis antimicrobiana en la cesárea y para el parto vaginal la plantean como opcional en los casos de alto riesgo de endocarditis, como en la paciente con válvulas artificiales, con antecedente de endocarditis o con enfermedad cianosante compleja. (10)

Sin embargo debido a la simplicidad, lo inocuo de la profilaxis y lo impredecible de las complicaciones del parto como desgarros grado IV, cateterización uretral en pacientes con bacteriuria inadvertida, etc., los obstetras y cardiólogos la recomiendan en todos los casos de enfermedad valvular adquirida o congénita. (1-2, 4-5), de igual manera es lo sugerido por el grupo de trabajo en cardiopatías y embarazo del Hospital San Vicente de Paúl.

La profilaxis se realiza con ampicilina 2 grs I.V ó I.M mas gentamicina $1.5 \mathrm{mg} / \mathrm{kg} 1 . \mathrm{V}$, en los 30 minutos antes del parto y una segunda dosis de ampicilina de 1 gr a las seis horas. Si hay alergia a la ampicilina se usa vancomicina.

\section{Agradecimientos}

Al Doctor Bernardo Agudelo, Ginecólogo y Obstetra Profesor del Departamento de Ginecología y Obstetricia de la Universidad de Antioquia, por su apoyo y revisión del manuscrito.

\section{Glosario}

Cardiopatía: Enfermedad del corazón.

Síndrome de Eisenmerg: Comunicación intracardíaca o de grandes vasos que cursa con elevación de la presión pulmonar.

Congénita: Hereditario, de nacimiento.

Cardiomiopatía: Enfermedad del miocardio. 
Retraso en el crecimiento intrauterino (RCIU): Crecimiento intrauterino, por debajo del percentil 5.

Shunt: Palabra inglesa, que se usa para expresar una desviación del flujo sanguíneo o por una vía alterna.
Profilaxis: Acción que se realiza, con el fin de prevenir una enfermedad.

Hipercoagulabilidad: Estado hemostático, que predispone a la coagulación venosa y/o arterial.

\section{BIBLIOGRAFIA}

1. Cunningham F, Mac Donald P, Gant N. Enfermedad cardiovascular. En Williams obstetricia. Editorial Panamericana. 20a edición, 1997; 1003-1023.

2. Oakley C, Elder M. Overview. En Heart disease in pregnancy. BMJ publishing group, 1997; 1-5.

3. Vásquez N. Cardiopatía y embarazo en el HUSVP en 1998. (Comunicación personal).

4. Shabetai R. Cardiac disease. En Creasy/Resnik. Maternal fetal medicine. W.B saunders company, 4th edition. P. 793-820.

5. Bonow et al. Management of valvular disease in pregnancy. En ACC/AHA task force report. JACC 1998; 32(5): 1545-1550.

6. Mestroni L, Miani D, Lenarda D, et al. Clinical and pathologic study of familial dilated cardiomyopathy. Am J Cardiol. 1990; 65: 1449. 1453
7. Sullivan, J; Ramanatha, K. Management of medical problems in pregnancy. Severe cardiac disease. NEJM. 1985; 304-308.

8. Arias, F. Cardiopatía y embarazo. En Guía práctica para el embarazo y el parto de alto riesgo. Harcourt Brace, 2a edición. 1997; P. $217-$ 233.

9. Oakley, C. Management of labor and delivery in the high risk patient. En Heart disease in pregnancy. BMJ publishing group, 1997; P. 375379.

10. Dajani A, Taubert K, Wilson W, et al. Prevention of bacterial endocarditis. Recomendations by the American heart association. JAMA. 1997. 277; 22: $1794-1801$. 